\section{ON SETTING UP A HEART TRANSPLANT PROGRAMME AT RAMATHIBODI HOSPITAL, BANGKOK, THAILAND}

Teerapat Yingchoncharoen. Ramathibodi Hospital and Mahidol University, Bangkok, Thailand

10.1136/heartasia-2019-apahff.3

The first heart transplant (HTx) programmes in Asia began in Taiwan and Thailand in $1987 .^{1}$ Since then, HTx has been accepted as a standard treatment for end-stage heart failure in Thailand. Currently, there are at least 10 Asian countries that have experience in HTx operation ${ }^{2}$ but few Asian countries (Taiwan, Korea and Japan) had more than 30 cases annually. ${ }^{3}$ The total number of HTx in Thailand during 2012-2017 ranged from 12-24 cases. There are five institutions in Bangkok that perform cardiac transplantation; namely, Chulalongkorn Hospital, Siriraj Hospital, Rajvithi Hospital, Bumrungrad Hospital and, in 2017, Ramathibodi Hospital started a new HTx programme after approximately 2 years of preparation. Our first case was a young male patient with myocarditis and cardiogenic shock requiring extracorporeal membrane oxygenator (ECMO) support. The patient survived the HTx despite a complicated clinical course. During 2017-2018, our centre performed 5 cardiac transplantation (2 out of 5 had ECMO support before transplantation, 1 was a paediatric patient) with zero in-hospital mortality. We are now in the very early stage of starting the HTx programme and there is still a lot of room for improvement in our programme.

\section{REFERENCES}

1. Ongcharit C, Ongcharit P. Intrathoracic organ transplantation in Thailand. Transplant Proc 1998;30:3385-3386.

2. Krittayaphong $R$, Ariyachaipanich A. Heart transplant in Asia. Heart Fail Clin 2015:11:563-572.

3. Lee HY, Oh BH. Heart transplantation in Asia. Circ J 2017;81:617-621.

\section{UPDATES ON CARDIAC TRANSPLANT AND LVAD IMPLANTS ACROSS THE UK AND EUROPE}

Steven SL Tsui. Department of Cardiothoracic Surgery, Royal Papworth Hospital, Cambridge, UK

\subsection{6/heartasia-2019-apahff.4}

Whilst there has recently been unprecedented growth in heart transplants $(\mathrm{HTx})$ in North America, the number has been static or falling in most European countries. These have resulted in significant increases in the waiting times.

In the UK, an Urgent Heart Allocation Scheme has been in existence since 2001. With a growing number of heart failure patients on temporary mechanical circulatory support (MCS) devices, a Super Urgent category was introduced in 2016. So far, $\sim 15 \%$ of HTx in the UK are performed under this new category and the median waiting time has been $\approx 7$ days. Post-transplant 30 day survival has been reassuring. However, ongoing monitoring will be required to ensure effectiveness.

The other major development has been donation after circulatory death (DCD) HTx. To date, 100 DCD HTx have been performed worldwide, with 70 of these being in the UK.
Growing waiting lists have led to increased implantation of bridge-to-transplant left ventricular assist devices (LVAD). However, the extended waiting times for donor hearts in stable patients mean that patients being bridged are effectively having destination therapy by default.

Whilst destination therapy is approved in some countries, the available evidence has not been accepted by other countries. The Swedish Evaluation of LVAD as Permanent Treatment in End-stage Heart Failure (SweVAD) is a prospective randomised study comparing LVAD therapy with optimal medical therapy. Recruitment commenced in 2016 with the aim of randomising 74 patients.

Outcomes and adverse events associated with implantable MCS will further improve as new devices using novel pumping mechanisms with lower shear stress are in development to address inherent limitations of current devices.

\section{OPTIMISING HEART FAILURE CARE BY MULTI- DISCIPLINARY HEART FAILURE CLINIC (MHFC)}

Kevin KH Kam, Ada Yu, Erik Fung, Alex PW Lee. Division of Cardiology, Department of Medicine and Therapeutics, Prince of Wales Hospital and The Chinese University of Hong Kong, Hong Kong SAR

\subsection{6/heartasia-2019-apahff.5}

According to the Hospital Authority's Statistical Report 2015-2016, heart failure (HF) was the leading cause of admission in Cardiology. The total number of HF admissions have increased by $14 \%$ in 10 years. Moreover, increasing frequency of acute decompensated $\mathrm{HF}$ has resulted in higher rates of hospitalisation and mortality. It is recognised that the 30 day readmission rate can be $25 \%-50 \%$ following hospital discharge. ${ }^{1}$ Current guideline recommends that HF patients should be managed by a multi-disciplinary care team to reduce HF-related hospitalisation. ${ }^{2}$ In fact, the establishment of multi-disciplinary heart failure clinics have been associated with reduction in rates of readmission ${ }^{3-5}$ and allcause mortality. ${ }^{5}$

In June 2017, we launched the Multi-disciplinary Heart Failure Clinic (MHFC) at Prince of Wales Hospital, Shatin, with two dedicated clinic sessions per week. Self-ambulatory patients in HF with reduced ejection fraction or HFREF (EF $\leq 40 \%$ ) and New York Heart Association (NYHA) class II-IV who had recent HF-related hospitalisation were followed. Education on self-monitoring of symptoms, fluid restriction and medication adherence were done by a dedicated HF nurse. Subsequent optimisation of guidelinedirected medical therapy was done by the cardiologist. At 6 month follow-up, NYHA class improved from I to II in the majority of patients (figure $1 ; \mathrm{p}=0.029$ ). In addition, the 30 day readmission rate decreased from $68 \%$ to $12 \%$ (figure $2 ; \mathrm{p}<0.05$ ).

In conclusion, the establishment of MHFC can improve HF-related hospitalisation and patients' symptomatology. Our local study echoes the findings of a recent meta-analysis. ${ }^{5}$

\section{REFERENCES}

1. Adib-Hajbaghery $M$, Maghaminejad $F$, Abbasi A. The role of continuous care in reducing readmission for patients with heart failure. J Caring Sci 2013;2:255267.

2. Ponikowski $P$, Voors $A A$, Anker SD, Bueno $H$, Cleland JGF, Coats AJS, Falk V González-Juanatey JR, Harjola VP, Jankowska EA, Jessup M, Linde C, 